\title{
Fracture of Be and its alloys - Webb Space Telescope revisited
}

\author{
Muhammad Musaddique Ali Rafique \\ Eastern Engineering Solutions Inc. 77 Massachusetts Ave, Cambridge MA \\ 02139, USA
}

\begin{abstract}
NASA/ESA/CSA joint venture James Webb Space Telescope is about to be launched. It is hypothesized to operate in near-infrared range. It is also hypothesized to unveil early star formation, galaxies, and universe due to its orbit, point in orbit and orbital motion. It has been under manufacturing for over 20 years at a staggering cost of 10 billion US dollars (most expensive scientific experiment in history). Beryllium (Be) is chosen to be element for construction of its main mirrors due to its high stiffness, low density, low linear coefficient of thermal expansion $(\alpha)$ in cryogenics and high thermal conductivity. It is followed by gold $\mathrm{Au})$ layer deposition on its $(\mathrm{Be})$ surface to enhance its sensitivity towards infrared radiation as later is hypothesized to bear superior properties. However, serious mistakes have been made in selecting this material for this application. Owing to its crystal structure (hexagonal close packed (hcp)), slip planes (basal, prismatic and pyramidal) and mechanisms of their activation, Be necessitates easy fracture at cryogenic temperature. It has anisotropic properties and prone to transverse fracture under tensile loading. Furthermore, its ductile to brittle transition temperature is very low making it entirely unsuitable for such an application. It is one of most expensive metals on planet. This study constitutes revisiting these fundamental properties and mechanisms which were entirely ignored during materials selection thus rendering whole project useless.
\end{abstract}

Keywords: Crystal structure, hexagonal close packed, slip planes, anisotropy, fracture 


\section{Introduction}

NASA/ESA/CSA's James Webb telescope is about to be launched to its intended orbit on single barrel three stage pencil rocket from south France. This project is claimed to be most sophisticated in history of science and thus have suffered various delays owing to its complexity, sophistication and difficulties encountered during design, material selection manufacturing and fabrication. Let aside, it had agglomerated to be of 10 billion $\$$ - most expensive experiment in history of science. No record exists about reverse calculations of intended orbit, payload deployed in intended orbit, its relationship with trajectory till point of launch on earth (recently shifted with a strong assumption that it will not affect any of afore and after-mentioned), mechanical design, architecture, materials selection, and manufacturing based on this. There were already open questions and now a closer look at material selection and its properties in proposed conditions have revealed even more bigger problems. Fundamental materials properties and selection criteria are entirely ignored so much so that it is very probable that this most expensive assembly will split into pieces during ascent inside or outside of faring of rocket. This study critically examines this and highlight fundamental mechanisms (slip system) which crucially dictate material selection, manufacturing, finishing, assembly, and application.

\section{Brittleness, crystallography, deformation, and fracture of Beryllium}

Beryllium is a hexagonal close packed (hcp) crystal structure metal. It is brittle at room temperature [1] and its brittleness increases as temperature is lowered [2]. It has three main slip systems (0001), $\{1 \overline{1} 00\}$ and $\{1 \overline{1} 0 X\}$ namely basal, prismatic, and pyramidal and there is only one slip direction $\langle 11 \overline{2} 0\rangle[3,4](\mathrm{Fig}-1)$. 


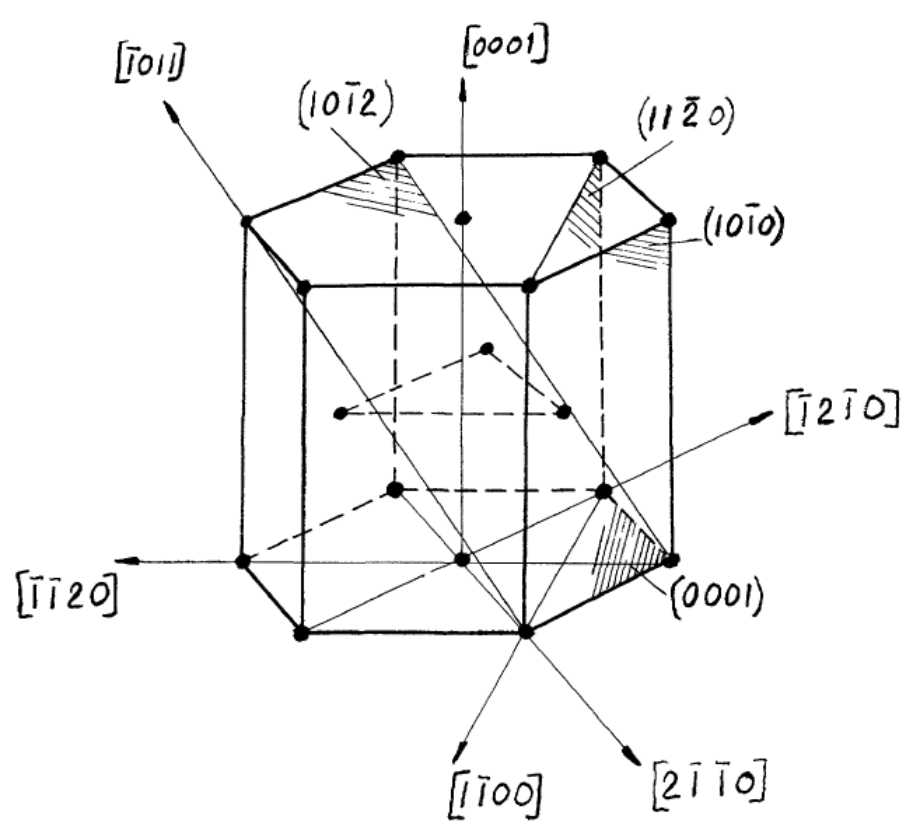

Fig - 1: Crystallographic planes and directions in a hcp lattice [1]

Mostly, under normal room temperature conditions and somewhat zero and near zero temperatures, basal plane is active and responsible for plasticity and fracture of this metal ( $5 \sigma_{b}=\sigma_{p}$ i.e. 5 times the stress at basal plane is required to start slip on prismatic plane. Typical $\sigma_{b}=1.4 \mathrm{Kg} / \mathrm{mm}^{2}$ ). However, at moderately high temperature, prismatic slip system is activated and result in deformation and fracture of metal while pyramidal only become active at very high temperatures [5]. This is very quick, and metal fails in brittle manner. Since application is cryogenic and involve thermal cycling, most likely this will happen. Other deformation mechanisms such as twinning, and cleavage are also activated under certain conditions. Twining plane is found out to be $\{11 \overline{2} 0\}$ and twining occurs by shear along $\langle 11 \overline{1} 0\rangle$ direction. Above $400^{\circ} \mathrm{C}$ two more twining planes are found to activate shear namely $\{10 \overline{1} 1\}$ and $\{10 \overline{1} 3\}$. Cleavage is found to occur primarily along basal plane (0001) and along secondary prism planes $\{1 \overline{1} 20\}$. Cleavage along twinning planes has also been observed. Role of c/a ratio for deformation modes and slip mechanisms reveal that Be lies in the middle of metals with hcp structure and found to be most brittle with well defined modes and slip mechanisms. For it, at its c/a ratio, tendency to slip by prismatic slip is high (despite high critical resolved shear stress) rendering metal less ductile and more brittle. It also offers much higher friction stress for motion of dislocations on basal plane as compared to its counterparts ( $\mathrm{Zn}$ or $\mathrm{Cd}$ ) (undesirable). A friction stress is due to 
lattice defects, impurity atoms, precipitates, and Peierls-Nabarro force of lattice itself. A high Peierls-Nabarro force implies inherent high slip resistance. Impurities in solid solutions can be reduced by purification of metals (inapplicable for Be (by condensation)) [6-8]). Thus, brittleness of Be is unavoidable. Another important factor which determines the inherent brittleness of Be is its very low value of Poisson Ratio $(<0.1)$ [9]. Decreasing grain size imparts slight ductility in Be. However, it has its own extrinsic (process dependent) limitations. Alloying seems not be affect ductility. Only limited enhancement in strength is achieved.

\section{Ductile to brittle transition temperature (DBTT)}

Ductile to brittle temperature of Be is shown to be a function of composition and thermal treatment (temperature range) [10]. For a commercially pure grade, it is $100-200^{\circ} \mathrm{C}[11]$. Below figure (Fig -2$)$ shows the variation of UTS, elongation and reduction in area for a wrought, fine grained, high purity beryllium product over a temperature range of $300-1000^{\circ} \mathrm{C}$ [11]. It can be easily observed that UTS which is a measure of strength not stiffness decreases as temperature increases. Elongation (usually represented as percentage) and reduction in area (which is measure of former) increase with increase in temperature. This is due to ability of material to toughen at the expense of strength.

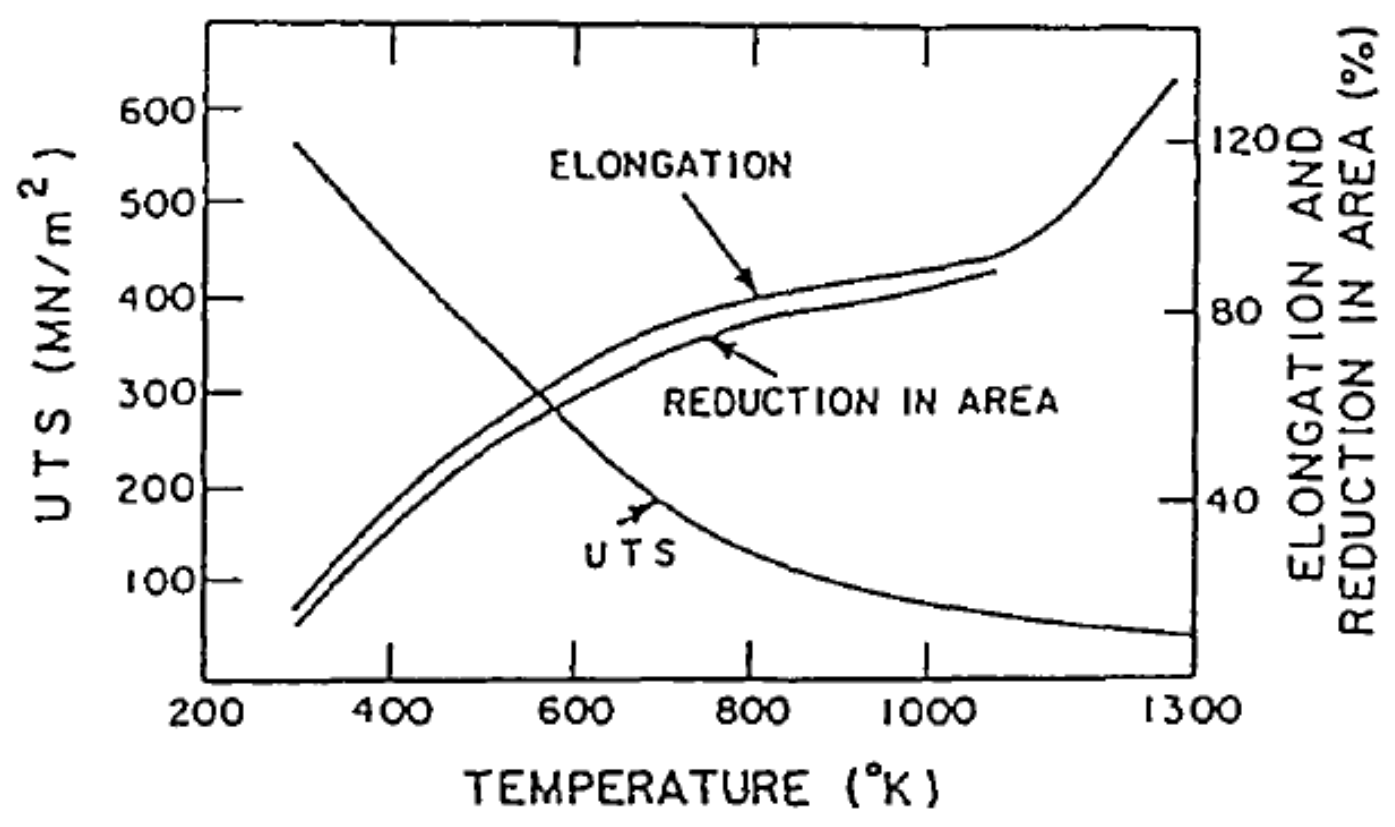

Fig-2: Temperature dependence of mechanical properties for a fine grained high purity product. [10] 
This DBTT is a strong function of strain rate [12]. Following two figures (Fig 3 and 4), shows effect of two different strain rates namely 0.001 and $1 \mathrm{~s}^{-1}$ on DBTT of various Be samples. In both cases, true strain, or reduction in sample height at which failure occurs is plotted against temperature from $1000^{\circ} \mathrm{C}$ (upper limit) to DBTT.

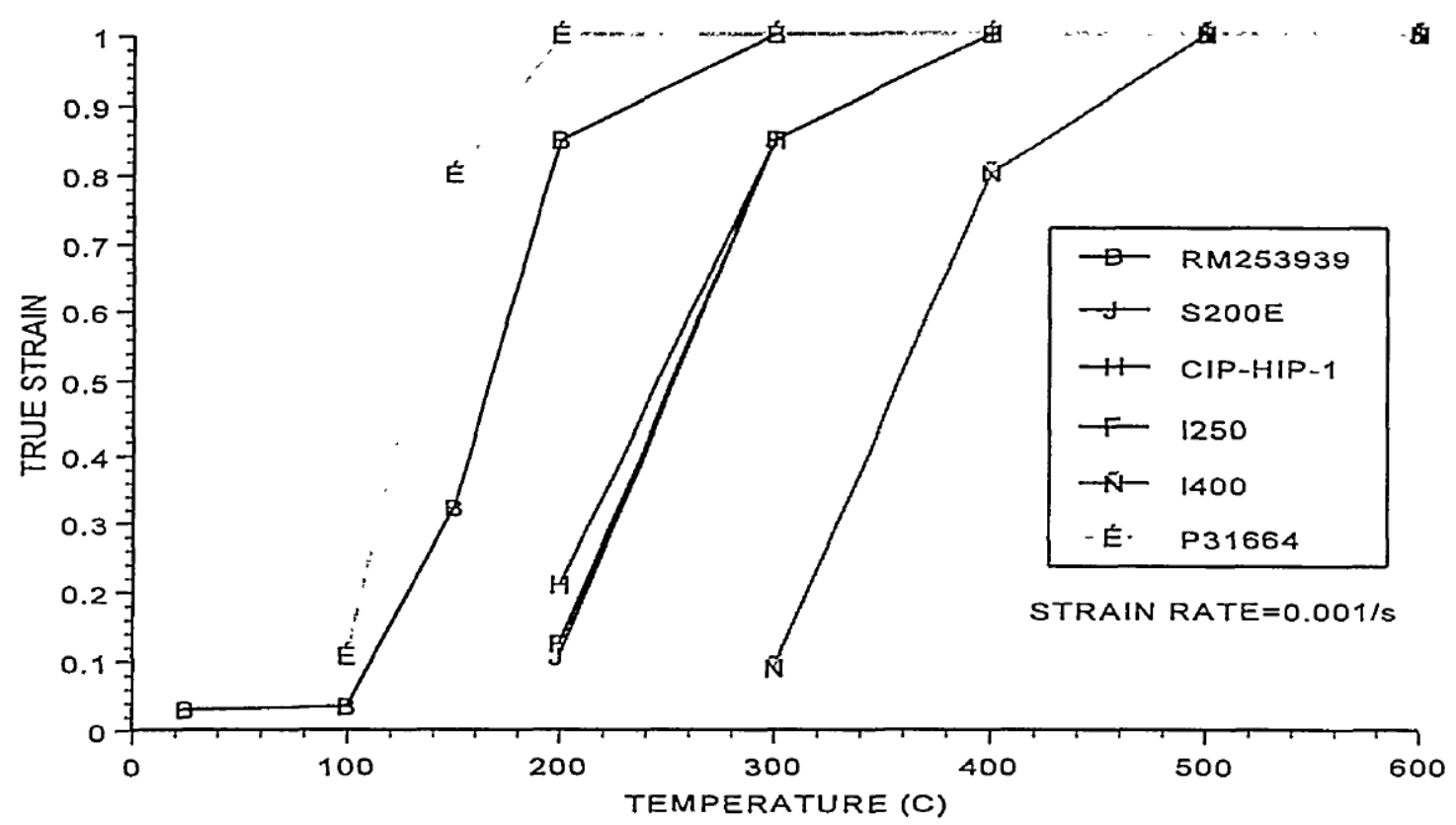

Fig - 3: True strain as a function of temperature at strain rate of $0.001 / \mathrm{s}$

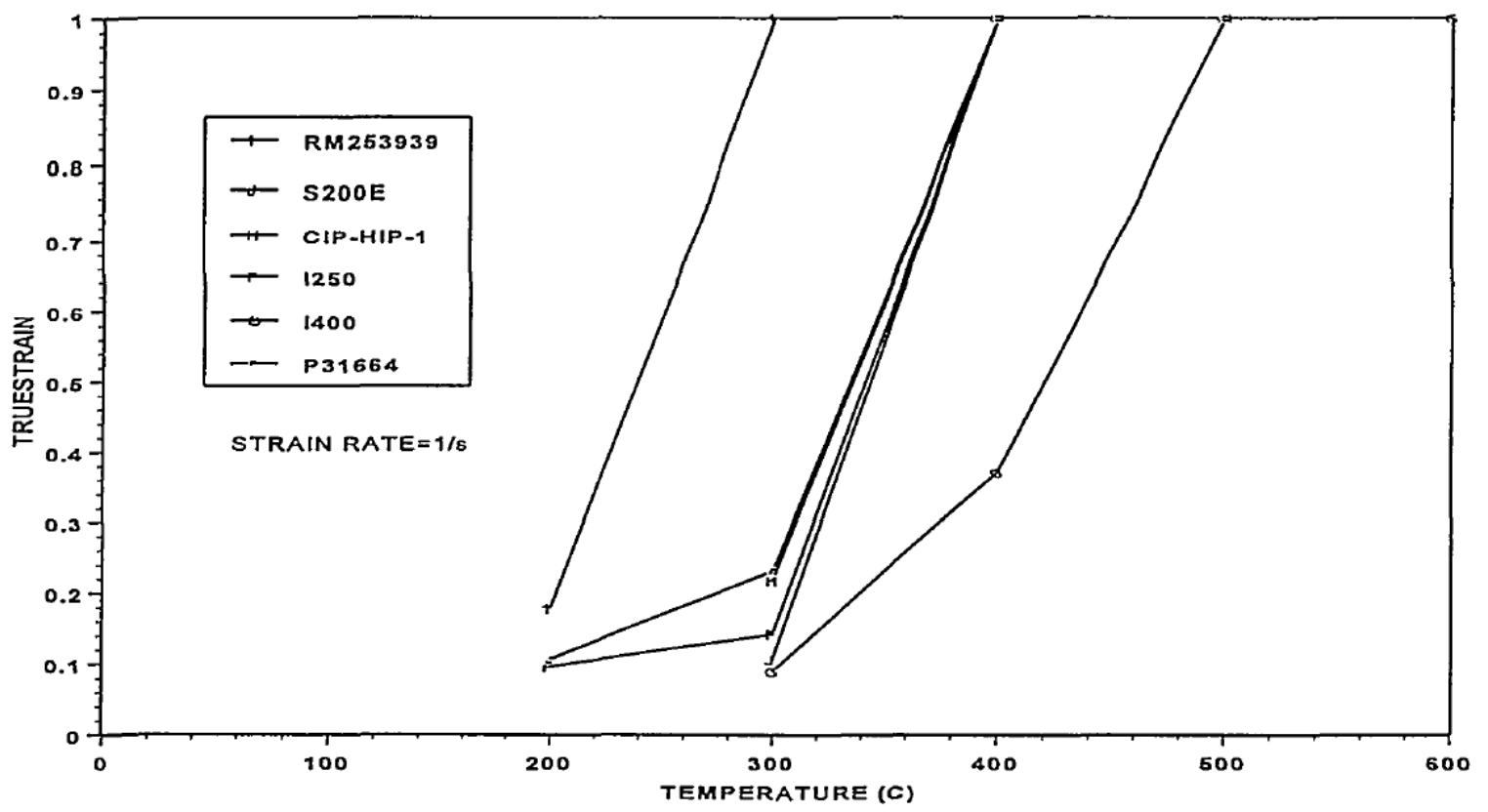

Fig - 4: True strain as a function of temperature at strain rate of $1 / \mathrm{s}$ 
It can be easily inferred all materials tested at 0.001 / s strain rate failed from tensile hoop stresses and cracked along the compression specimen surface. DBTT range is from 400 to $100^{\circ} \mathrm{C}$. Strongest material exhibits highest DBTT [2, 12, 13]. In case of figure 4 (strain rate $1 / \mathrm{s}$ ), lower limit of DBBT is raised to 200 with same upper limit of 400C. Fracture mode also slightly changed from tensile hoop stress fracture to adiabatic shear band failure at $45^{\circ}$. Yield point variations were observed in various grades bearing various compositions. Flow stress and shape of flow curves varies considerably with grades due to variation in alloy content, size and distribution of $\mathrm{BeO}$ particles, aging precipitates, and grain size (Fig - 5).

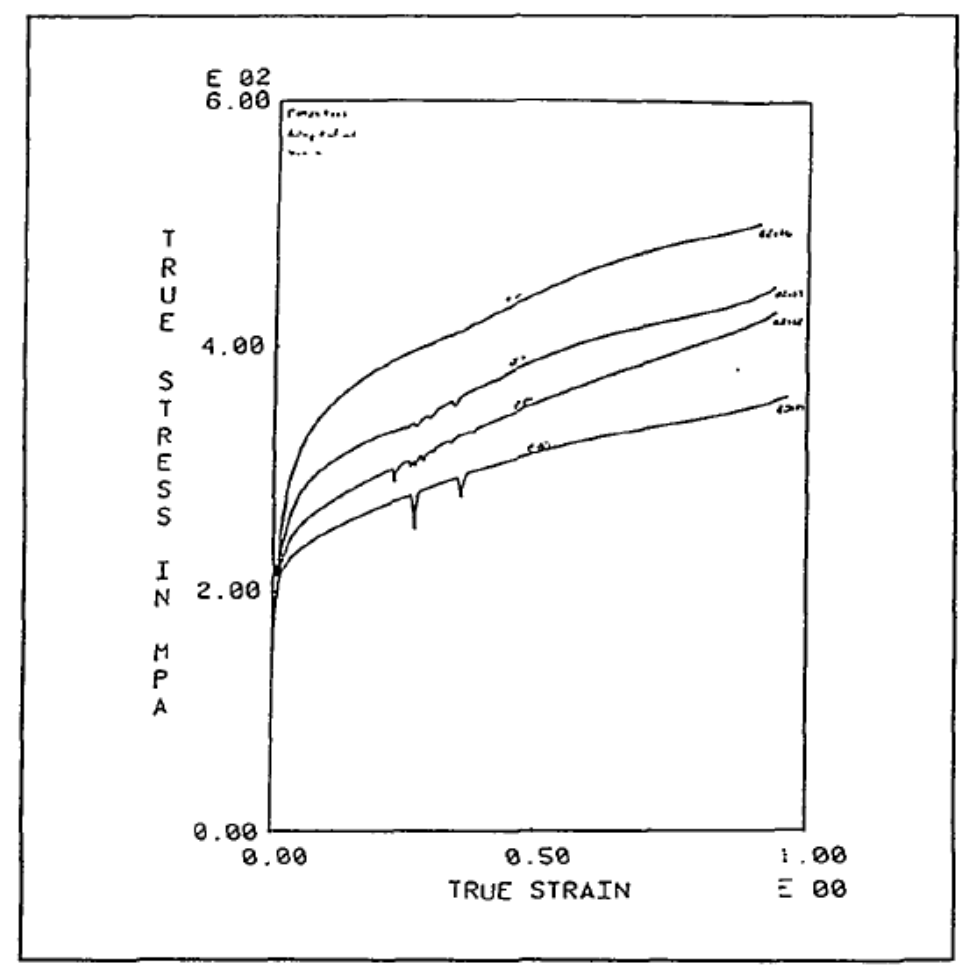

Fig - 5: General characteristic of serrated flow observed at $500^{\circ} \mathrm{C}$

\subsection{Anisotropy}

DBTT is highly anisotropic [13]. This is accounted by measuring its value in a rolled sheet subjected to tensile testing. It showed inferior tensile ductility in transverse direction to those in longitudinal direction in the temperature range of $100-400^{\circ} \mathrm{C}$. Even annealing after words does not improve much (Fig -6$)$. 
Fig - 6: Tensile stress strain curves at $300^{\circ} \mathrm{C}$ for rolled $\mathrm{Be}$ sheets annealed at $900^{\circ} \mathrm{C}$

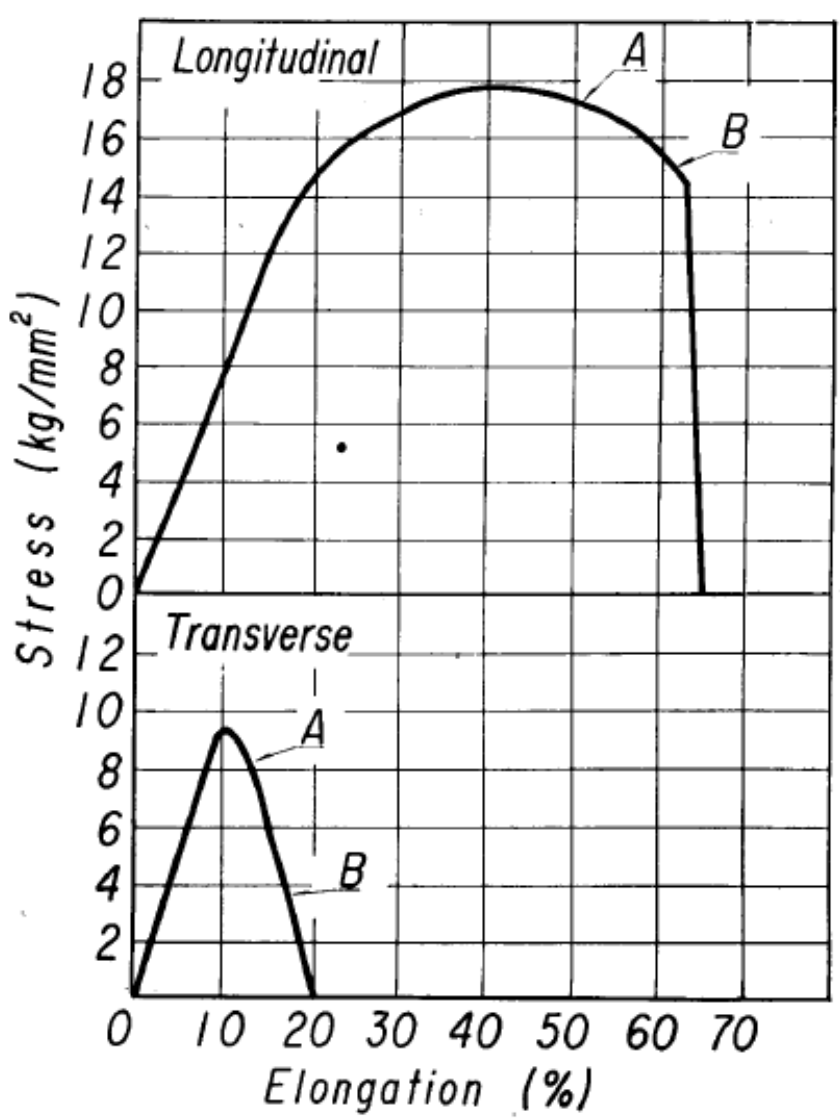

It can be easily observed that mechanical properties (Y.S, UTS and Percentage elongation) are considerably low along transverse direction. Following figure (Fig - 7) shows variation of yield strength with testing temperature for Be Sheets followed by their microstructures in Fig $-8(a, b)$.

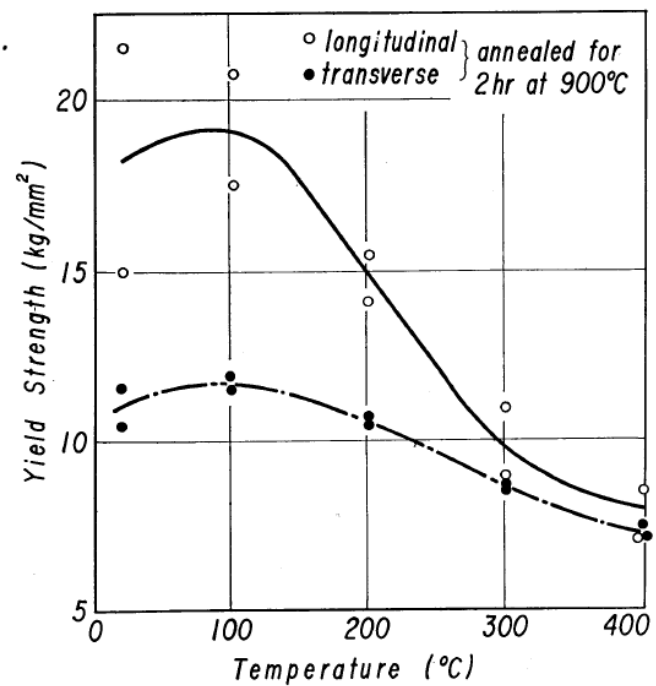

Fig - 7: Variation of yield strength with testing temperature for Be Sheets 


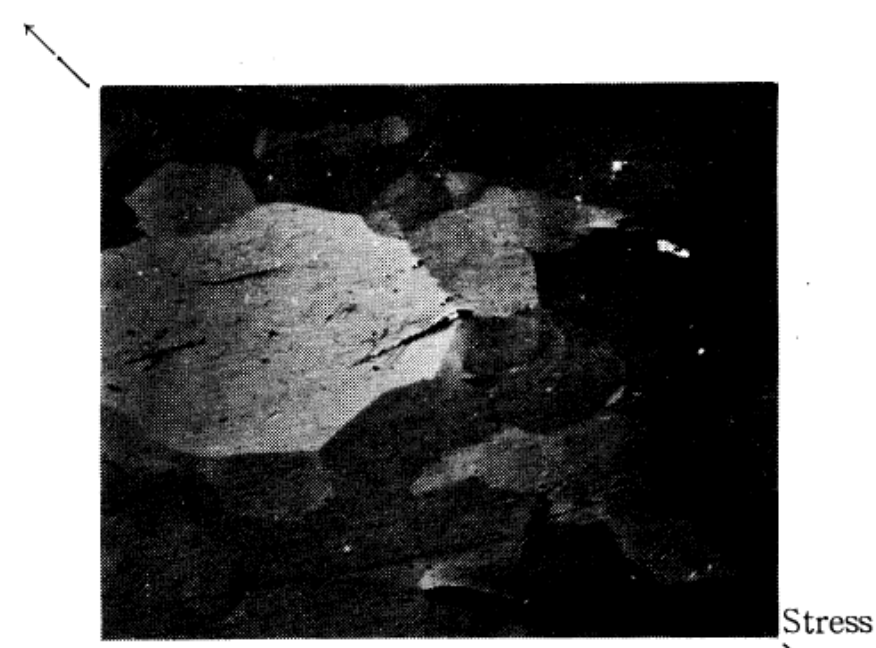

(a) Longitudinal specimen

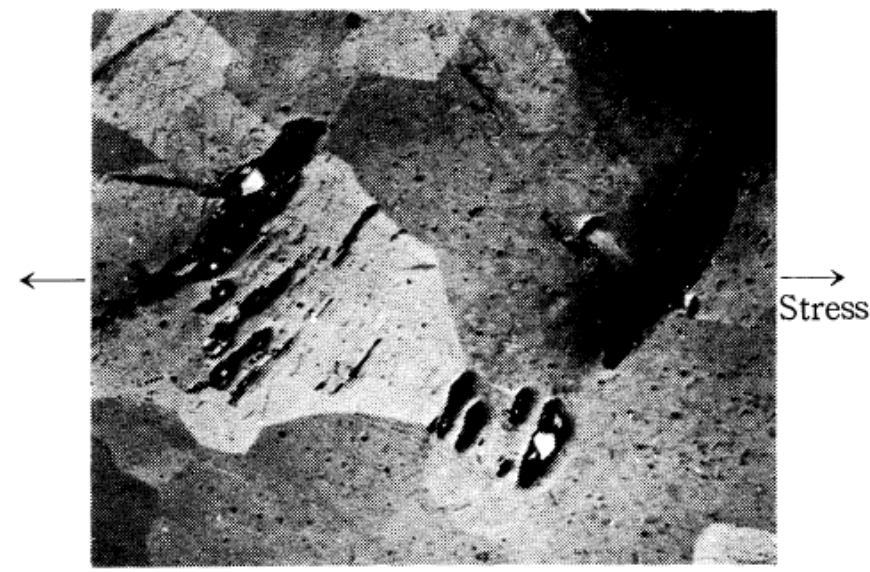

(b) Transverse specimen

Fig - 8 (a, b): Optical (Polarized) light micrographs of specimens with two different rolling directions.

Trans granular cracks are easily observable in specimens which were strained till point A in Fig 6.

\section{Conclusions}

Following conclusion may be drawn

1. Beryllium is a hexagonal close packed (hcp) crystal structure metal. It is brittle at room temperature and its brittleness increases as temperature is lowered.

2. There are certain preferred planes along which deformation occurs by virtue of slip mechanisms. These are basal plane (0001), prismatic $\{1 \overline{1} 00\}$ and pyramidal $\{1 \overline{1} 0 X\}$. Slip direction being one only $\langle 11 \overline{2} 0\rangle$. 
3. Mostly slip occurs along basal plane, occasionally along prismatic at higher temperature and higher shear stress value (usually when stress for this (prismatic stress) exceeds critical resolved shear stress at basal plane $\left(\sigma_{b}=\right.$ $\left.1.4 \mathrm{Kg} / \mathrm{mm}^{2}\right)$ ) while pyramidal plane is activated at very high temperatures only.

4. DBTT is highly anisotropic. Its lower value is observed along transverse direction with trnsgranular cracks as compared to longitudinal direction.

\section{References}

1. Lagerberg, G., Metallurgical viewpoints on the brittleness of beryllium. 1960: Sweden. p. 24.

2. LOtt, L.A. LITERATURE SURVEY: PHYSICAL PROPERTIES OF LITHIUM AND BERYLLIUM AT LOW TEMPERATURES. 1967.

3. Petch, N.J. and E. Wright, The Plasticity and Cleavage of Polycrystalline Beryllium. II. The Cleavage Strength and Ductility Transition Temperature. Proceedings of the Royal Society of London. Series A, Mathematical and Physical Sciences, 1980. 370(1740): p. 29-39.

4. Z Zhong, J.M., et al., Micro-yield behavior and mechanism of beryllium metal. 2004. 14: p. 16371641.

5. Goldberg, A., et al., Physical Metallurgy of Beryllium, in Beryllium Chemistry and Processing, E.E. Vidal, et al., Editors. 2009, ASM International. p. 0.

6. Rengstorff, G.W.P., et al., High-purity Metals. 1966: Defense Metals Information Center, Battelle Memorial Institute.

7. Commission, U.S.A.E., U.S.E. Research, and D. Administration, Nuclear Science Abstracts. 1959: Oak Ridge Directed Operations, Technical Information Division.

8. Goldberg, A., Beryllium Manufacturing Processes. 2006: United States.

9. Pugh, S., XCII. Relations between the elastic moduli and the plastic properties of polycrystalline pure metals. The London, Edinburgh, and Dublin Philosophical Magazine and Journal of Science, 1954. 45(367): p. 823-843.

10. Abeln, S.P., R. Field, and M.C. Mataya, Elevated temperature stress strain behavior of beryllium powder product. 1995: United States. p. 33.

11. Henshall, G.A., S.G. Torres, and J.E. Hanafee, The elevated temperature tensile properties of S200E commercially pure beryllium. 1995: United States. p. 106-121.

12. Conrad, H. and F.W. Cooke, The effects of temperature and strain rate on the strength of beryllium sheet. Metallurgical Transactions, 1971. 2(5): p. 1307-1313.

13. Tsuya, K., Anisotropy of the Ductile-Brittle Transition Behavior of Beryllium Sheets. Journal of the Japan Institute of Metals, 1966. 30(4): p. 372-376. 